\title{
Cucurbit[7]uril-Anchored Porphyrin-Based Multifunctional Molecular Platform for Photodynamic Antimicrobial and Cancer Therapy
}

\author{
Melis Özkan, ${ }^{\dagger}$ Yogesh Kumar, $^{\dagger, \ddagger}$ Yagmur Keser, $^{\dagger}$ Seyed E. Hadi, ${ }^{\dagger}$ and Dönüs Tuncel ${ }^{*}, \dagger \oplus$ \\ ${ }^{\dagger}$ Institute of Materials Science and Nanotechnology, National Nanotechnology Research Center (UNAM), Bilkent University, \\ 06800 Ankara, Turkey \\ ${ }^{\ddagger}$ Department of Chemistry, Bilkent University, 06800 Ankara, Turkey
}

\section{Supporting Information}

\begin{abstract}
Here we report a photoactive supramolecular assembly that is multifunctional and constructed by covalently linking four receptor molecules (cucurbit[7] uril) to a porphyrin derivative with suitable linkers. While this molecular platform serves very efficiently as a light-triggered broad-spectrum antibacterial agent, owing to its negligible dark cytotoxicity and the presence of host molecules (CB7), it can also be utilized as a vehicle to carry drug molecules for a combined chemo and photodynamic cancer therapy.
\end{abstract}

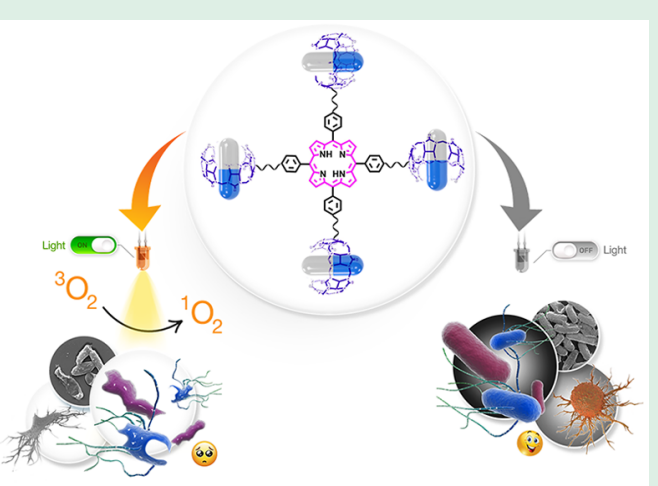

KEYWORDS: photodynamic antimicrobial therapy, cucurbituril, multifunctional molecular platform, chemo-photodynamic dual cancer therapy, porphyrin, photosensitizer, drug delivery

$\mathrm{P}$ hotodynamic therapy (PDT) has been receiving an increasing attention both to treat certain types of cancers and infectious diseases caused by bacteria. ${ }^{1-3}$ Reactive oxygen species (ROS) and singlet oxygens are generated from the molecular oxygens under light with the assistance of a suitable photosensitizer. Singlet oxygens, which are the essential component of the PDT, are highly reactive species with an ability of killing cancer cells and pathogens. PDT is proven to be a very effective method especially in the inactivation of bacteria because the bacteria have been developing a resistance against the antibiotics, and this, in turn, makes the treatment with drugs less efficient. ${ }^{4,5}$ The usage of PDT in cancer therapy also quite appealing due to the unwanted side effects of the conventional methods on the body.

In PDT, one of the most important components is the photosensitizer, which should ideally be water-soluble with negligible dark toxicity, but it should be cytotoxic only when it is irradiated by a suitable light source. In this regard, porphyrin-based photosensitizers are quite appealing owing to their tunable properties. ${ }^{6,7}$ For instance, their water solubility and photophysical properties can be altered by conjugating various hydrophilic and bulky functional groups to porphyrin cores. 8

Recently, cucurbit[n]uril (CB) containing supramolecules have also been employed as photosensitizers in photodynamic antibacterial therapy. ${ }^{10-14}$ It was reported that while cationic porphyrin derivatives exhibit high cytotoxicity toward bacteria in the dark, their toxicity can be reduced by inclusion complex formation with $\mathrm{CB}$ homologues. Thus, the toxicity can be switched on and off at will as the complex becomes highly toxic upon light treatment. ${ }^{11,12}$ We have also reported recently a [5]rotaxane-based photosensitizer for very efficient photodynamic antibacterial and cancer therapies. ${ }^{15}$

CBs are multifunctional macrocycles with a hydrophobic cavity and carbonyl containing hydrophilic portals. They can form inclusion complexes with various guests having appropriate sizes and shapes including dyes, drugs, and peptides. ${ }^{16,17}$ Supramolecular assemblies and nanostructures have been constructed either through host-guest complexation ability of CBs with a variety of molecules or conjugating CBs through covalent bonds to various molecules. ${ }^{18,19}$ While there are many examples in the literature reporting the complexes of porphyrin derivatives with cucurbituril homologues, ${ }^{19-21}$ the examples are quite rare on the covalently linked CB-porphyrin based molecular platforms to bring together the best words of these two remarkable molecules. $^{22-24}$

Although chemotherapy remains to be one of the important clinical antitumor approaches, some unwanted side effects caused by the drugs are a major issue. ${ }^{25}$ These problems can be

Received: August 23, 2019

Accepted: October 23, 2019

Published: October 23, 2019 
circumvented, and the diseases can be treated more efficiently, if multiple modalities such as chemo and photodynamic therapies are combined in one platform to bring out their synergistic effects. ${ }^{26,27}$

In this context, we report here a photoactive multifunctional molecular platform based on a porphyrin core decorated with four CB host molecules and employ this platform for multiple purposes. While the porphyrin core is responsible for the photodynamic effect, CBs can host the drug molecules for delivery. Consequently, this unique molecular platform has been proven to be an efficient, light-triggered broad-spectrum antibacterial agent by inhibiting growth of bacteria (both Gram-negative and Gram-positive). It can also be employed as a vehicle to carry anticancer drugs for a dual chemo and phototherapy of cancer making use of their synergistic effect.

We synthesized TTP-4CB7 by following the procedure reported in our previous paper. ${ }^{23}$ Scheme 1 shows its synthetic

Scheme 1. Synthesis of Cucurbit[7] uril-Anchored
Porphyrin Assembly (TPP-4CB7)

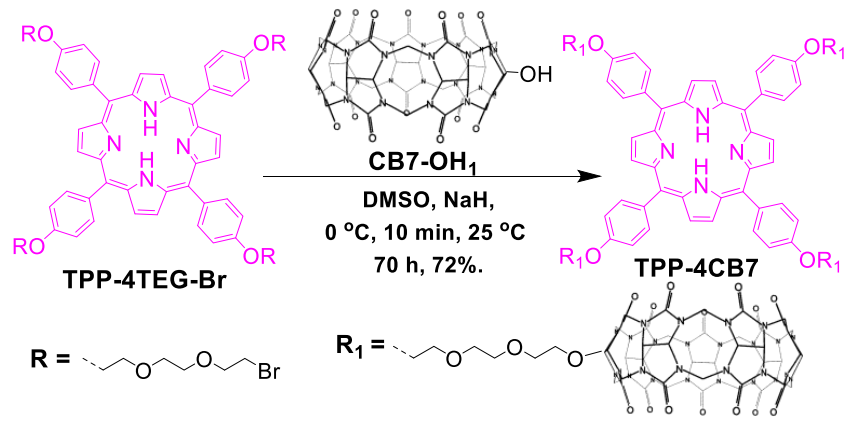

routes. The Supporting Information section provides some characterization data (Figures S1-S3). This molecular platform has a reasonable water solubility to carry out the biological assays.

UV-vis spectrum of TPP-4CB7 was recorded in water (Figure S3), which displayed an absorption peak at $435 \mathrm{~nm}$ (Soret band) and four peaks at 525, 565, 600, and $655 \mathrm{~nm}$ (Qband). Its fluorescent quantum yield was determined to be around $1 \%$. Its ability to produce singlet oxygen was studied by 2,7-dichlorofluorescein diacetate (DCFH-DA) assay ${ }^{15}$ under white light $\left(1 \mathrm{~mW} / \mathrm{cm}^{2}\right.$ light fluence). DCFH-DA was first hydrolyzed into 2,7-dichlorofluorescin (DCFH) in the basic medium (Scheme 2). When TPP-4CB7 was mixed with nonfluorescent DCFH and irradiated with light, an emission band at around $523 \mathrm{~nm}$ appeared because of the oxidation of DCFH to fluorescent DCF with the generated singlet oxygen. This emission band intensity continued to increase by increasing the exposure time (Figure S4). These results indicate that TPP-4CB7 can generate ROS efficiently light with broad excitation wavelengths and low intensity.

By taking advantage of the ability of TPP-4CB7 to generate singlet oxygen, we next set out to make use of this photosensitizer in the antibacterial photodynamic therapy. Its antibacterial activity was probed both on Eschericha coli (E. coli) and Bacillus subtilis (B. subtilis), which are, respectively, Gram-negative and Gram-positive bacteria. The viability of bacteria was analyzed by evaluating the minimum inhibitory concentration (MIC) value. For this purpose, first E. coli was treated with varying concentration of TPP-4CB7, and the resulting suspensions were exposed with white light $(22 \mathrm{~mW} /$ $\mathrm{cm}^{2}, 120 \mathrm{~s}$ ). MIC was determined to be around $5.7 \mu \mathrm{M}$ under these conditions (Figures S5 and S6). Bacterial survival experiments were performed using $5.7 \mu \mathrm{M}$ TPP-4CB7 in the dark and under light exposure (Figures $1 \mathrm{~b}-\mathrm{e}, \mathrm{S} 7$, and S8). According to the colony counting results, more than 3-log reduction was observed in the number of colonies after incubating E. coli bacteria with $5.7 \mu \mathrm{M}$ concentration of TPP4CB7 and irradiating the suspension. Under the same condition but in the absence of light, the bacteria inhibition was insignificant (Figure $1 \mathrm{~b}$ ).

We also investigated the biocidal activity of TPP-4CB7 on B. subtilis bacteria by keeping the similar conditions (concentration of the photosensitizer, exposure time and intensity of the light) used for E. coli bacteria; in the dark, around $70 \%$ and with light exposure, $100 \%$ of bacteria were inactivated (Figures S9-S11). Observed relatively high dark toxicity of TPP-4CB7 against B. subtilis bacteria is not totally surprising as Gram-positive and Gram-negative bacteria have significant structural differences in their cell walls. Gramnegative bacteria show higher resistant to antibiotics than do Gram-positive bacteria due their protective outer membrane at their cell walls that prevents the penetration of antibiotics. On the other hand, antibiotics can more easily diffuse through the membrane of Gram-positive bacteria. ${ }^{28}$ Remarkable killing efficiency of TPP-4CB7 for both bacteria types proves its suitability as a promising broad-spectrum photoantibacterial agent.

To reveal the morphology E-coli bacteria after incubating with TPP-4CB7 in the dark and under light, scanning electron microscopy (SEM) was utilized to take their images (Figure 2 ). When bacteria were incubated with photosensitizer in the dark in the absence of light (Figure 2b), they preserved their rod-like shapes and their surfaces remained smooth without any damages. However, upon light treatment and in the presence of photosensitizer, the morphology of E. coli bacteria was totally changed (Figure $2 \mathrm{~d}$ ) compared to its light treated control group (Figure 2c). Their membranes were ruptured, and their cytoplasmic contents leaked out. To further understand the binding efficiency of $E$. coli bacteria with TPP-4CB7, they were incubated with TPP-4CB7 in the dark

Scheme 2. Mechanism of DCFH-DA to DCFH, Which Is Oxidized to Fluorescent DCF<smiles>CC(=O)Oc1cc2c(cc1Cl)C(c1ccccc1C(=O)O)c1cc(Cl)c(OC(C)=O)cc1O2</smiles>

2,7-dichlorofluorescein diacetate (DCFH-DA)

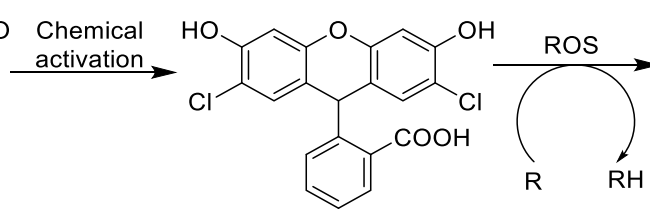

2,7-dichlorofluorescein (DCFH)<smiles>O=C(O)c1ccccc1-c1c2cc(Cl)c(=O)cc-2oc2cc(O)c(Cl)cc12</smiles>

2,7-dichlorofluorescein (DCF) 
(a)

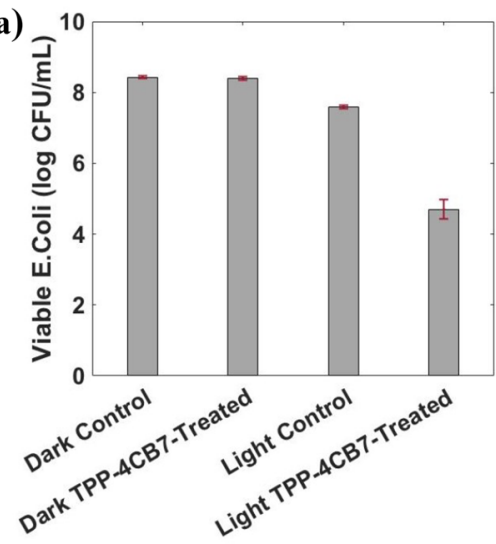

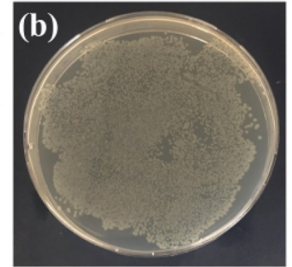
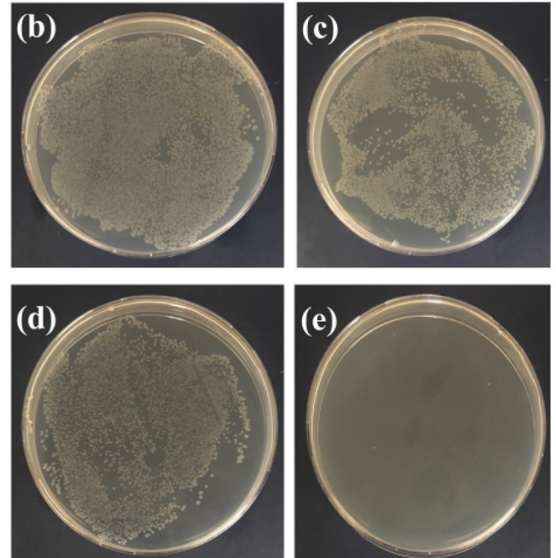

Figure 1. (a) Antibacterial activity of TPP-4CB7 tested on E. coli bacteria in the dark and under light (white light, $22 \mathrm{~mW} / \mathrm{cm}^{2}, 120 \mathrm{~s}$ ), the mean \pm standard deviation (SD) and error bars were calculated from the results of six different assays. Photographs of $E$. coli bacteria on agar plate, (b) $E$. coli only in the dark, (c) in the presence of TPP-4CB7 in the dark, (d) E. coli only under light and in the presence of TPP-4CB7 and (e) under light.
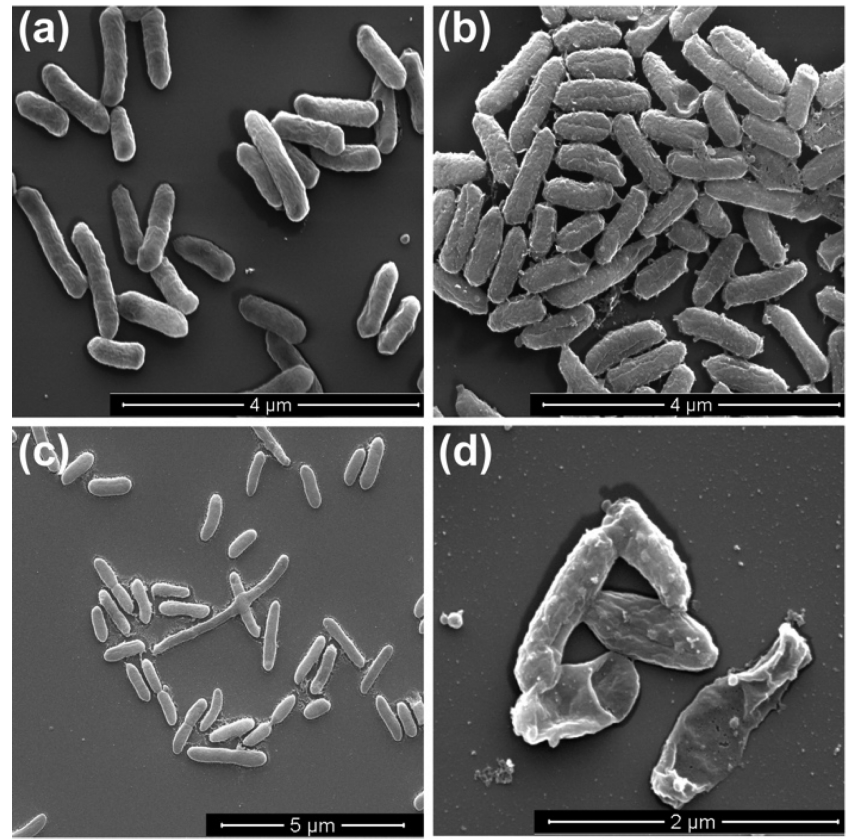

Figure 2. SEM images of (a) E. coli. only, no light, (b) TPP-4CB7 $(5.7 \mu \mathrm{M})$-treated E. coli bacteria, no light, (c) E. coli. bacteria only, with light exposure, (d) TPP-4CB7 $(5.7 \mu \mathrm{M})$ treated bacteria with light exposure (white light, $22 \mathrm{~mW} / \mathrm{cm}^{2}$ and $120 \mathrm{~s}$ ).

and under light, and their $\zeta$-potential values were measured (Figure S12). $\zeta$-Potential values of $E$. coli were shifted from -40 to $-23 \mathrm{mV}$ in the absence of light and from -44 to -29 $\mathrm{mV}$ with light exposure. These results support that there are effective interactions between bacteria and the photosensitizer.

We have conducted a MIC assay by incubating E. coli bacteria with methylene blue (MB) as a control drug experiment by keeping the conditions similar to the one used for our photosensitizer, TPP-4CB7. When the biocidal activities of the photosensitizer TPP-4CB7 are compared with $\mathrm{MB}$ in the dark and under light, it can be seen that $\mathrm{MB}$ shows high dark toxicity even at low concentration (MIC is around 4 $\mu \mathrm{M}$ ), but TPP-4CB7 is not toxic in the dark (Figure S5). When bacteria were treated both MB and TPP-4CB7 under light, MIC of the MB was slightly lower than TPP-4CB7, around $3.5 \mu \mathrm{M}$ and $5.7 \mu \mathrm{M}$, respectively (Figure S6). Although MIC of MB is slightly higher than TPP-4CB7 under light, TPP-4CB7 exhibits a negligible dark toxicity, which makes TPP-4CB7 an attractive photosensitizer for PDT.

We next investigated the in vitro cytotoxicity and photocytotoxicity of TPP-4CB7. For this purpose, MCF-7 breast cancer cell line was used, and the photosensitizer concentration range of $1-100 \mu \mathrm{M}$ was selected to evaluate the cell viability against TPP-4CB7 by MTT assays. In the absence of light, the value of half maximal inhibition concentration $\left(\mathrm{IC}_{50}\right)$ of TPP-4CB7 was determined as higher than $100 \mu \mathrm{M}$ (Figure 3a). However, upon light exposure even at the lowest concentration $(1 \mu \mathrm{M})$ of TPP-4CB7, around $25 \%$ of inhibition in the cell growth was achieved (Figure $3 b$ ). Inhibition rate continued to rise with gradual increase in the concentration of TPP-4CB7; 50\% decrease was observed at a relatively low concentration $(5.5 \mu \mathrm{M})$ (Figure $3 \mathrm{~b})$, and this rate reached to over $90 \%$ at the concentration of $100 \mu \mathrm{M}$. These in vitro cell assays show that under brief exposure and low fluence of light even at low concentration of TPP-4CB7, cell viability decreases substantially, indicating that TPP-4CB7 is a promising photosensitizer for PDT.

As a next step, we wanted to demonstrate the use of TPP4CB in the combined chemo and photodynamic therapy as CB7 molecules can complex with drugs molecules and can be used as a drug carrier. For this purpose, a well-known anticancer drug, doxorubicin (DOX), was chosen. Recently it was also shown by molecular modeling that ammonium and hydroxyl groups of DOX molecules complex with the carbonyl portal of CB7 through H-bonding. ${ }^{29,30}$ TPP-4CB7+DOX complexes were prepared by stirring DOX with TPP-4CB7 in DMEM. Cell viability studies were carried out through MTT assays using MCF-7 cells. The cells have been treated with varying concentration of TPP-4CB7, DOX, and TPP4CB7+DOX in the dark and upon light exposure. The incubation time was kept around $96 \mathrm{~h}$. Viability of the cells decreased by increasing doses of DOX in the dark, and similar results were observed under light (Figure 3a,b). The activity of DOX molecules was found to be slightly lower when they were carried by TPP-CB7 and in the absence of light exposure. However, $50 \%$ inhibition was achieved under light at $10 \mu \mathrm{M}$ DOX concentration in the presence of $4 \mu \mathrm{M}$ TPP-4CB7 compared to $50 \%$ inhibition caused by light treated DOX and 

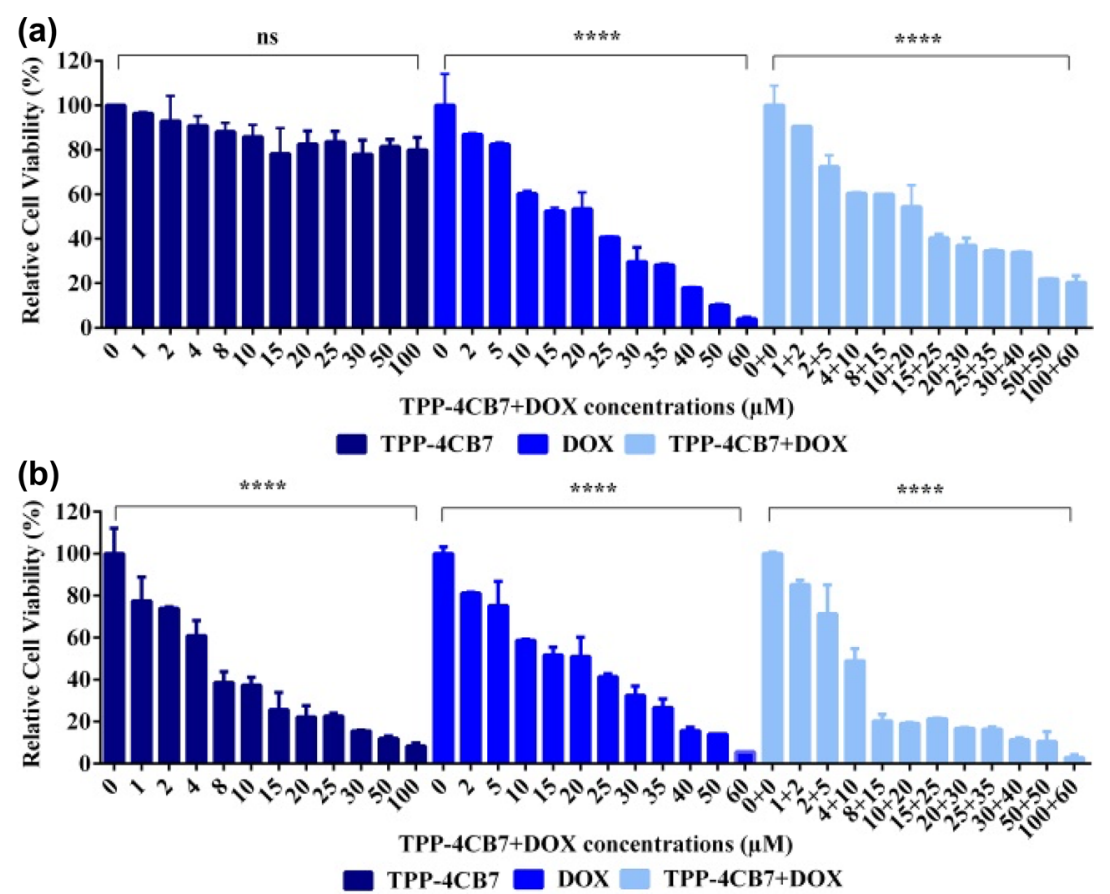

Figure 3. MTT assays of MCF-7 cells incubated with a number of different doses of the photosensitizer (TPP-4CB7), doxorubicin (DOX), and DOX-loaded TPP-4CB7, (a) no light and (b) upon light exposure (white light, $5 \mathrm{~min}, 20 \mathrm{~mW} / \mathrm{cm}^{2}$ ). MTT assays were normalized to the standard DMEM, and the results were analyzed as reported in the literature. ${ }^{15} * * * *, P<0.0001$; ns, nonsignificant.

TPP-4CB7 individually at the concentrations of $20 \mu \mathrm{M}$ and $5.5 \mu \mathrm{M}$, respectively. Furthermore, at the highest concentration, we observed almost a $100 \%$ inhibition in the cell growth. These results confirm that when the chemo and photodynamic effects are used in combination in one design, the treatment can be highly effective.

To gain insight on how DOX molecules are binding to CB7s, we have recorded a ${ }^{1} \mathrm{H}$ NMR spectrum of TPP-4CB7 with DOX, but the spectrum was not helpful as it is mainly dominated by CB7 peaks because each molecular platform contains four CB7 units. Accordingly, we have recorded ${ }^{1} \mathrm{H}$ NMR spectra of DOX with CB7. The spectra we obtained were similar to those reported in the literature. There were no significant changes in the chemical shifts for DOX protons in the presence of CB7 indicating the low binding constant of complexation. As suggested by Trabolsi et al. ${ }^{30}$ based on their computational modeling, ammonium and $\mathrm{OH}$ groups of $\mathrm{DOX}$ probably complex with carbonyl groups of $\mathrm{CBs}$ through $\mathrm{H}$ bonding.

We have also conducted ITC with TPP-4CB7, but the results were not satisfactory. Therefore, we studied the nature of the interaction between neat CB7 and DOX in the DMEM, medium that was also used for the cell assays (Figure S13). The results are not very satisfactory but still provide some insights. The binding stoichiometry suggested by ITC is around 1:0.85 with a binding constant of $7.36 \times 10^{5} \pm 2.45 \times$ $10^{5} \mathrm{M}^{-1}$.

We should also mention that although each molecular platform contains four CB7 units, in this work, the amount of DOX used was kept limited with a maximum ratio of TPP4 CB7 to DOX (1:1.5). It means that probably one DOX molecule is complexing with more than one CB7 unit of TPPCB7; this, in turn, causes a slow release of DOX drug molecules.
In conclusion, we reported here a multifunctional supramolecular photosensitizer that consists of multiple CB7 units that are available for a host-guest chemistry and a photoactive porphyrin core. While this photosensitizer exhibits no dark cytotoxicity toward Gram-negative bacteria (E. coli), it shows relatively high cytotoxicity on the Gram-positive bacteria ( $B$. subtilis). However, this photosensitizer becomes highly toxic toward both types of bacteria (E. coli and B. subtilis) upon light exposure. Even with short exposure time and low fluence of white light, complete inhibition of both bacteria growth (around 100\%) can be achieved; these results prove the potentiality of TPP-4CB7 as a broad-spectrum antibacterial agent. Although it shows no significant dark cytotoxicity against MCF7 cancer cell line even at the high doses of photosensitizer (with $\mathrm{IC}_{50}>100 \mu \mathrm{M}$ ), $50 \%$ of the cells were inactivated with a low dose of $5.5 \mu \mathrm{M}$ under white light. We have also demonstrated the application of this molecular platform in chemo-photodynamic dual cancer therapy using MCF-7 cells. Light-treated molecular platform (TPP-4CB7) loaded with DOX molecules inactivates the cells efficiently and shows the synergistic effects of PDT and chemotherapy.

\section{ASSOCIATED CONTENT}

\section{Supporting Information}

The Supporting Information is available free of charge on the ACS Publications website at DOI: 10.1021/acsabm.9b00763.

Experimental details, ${ }^{1} \mathrm{H}$ NMR, ESI-MS, UV-vis absorbance spectra of TPP-4CB7, PL spectra of DCF in the presence of TPP-4CB7 under continuous whitelight illumination, MIC assays, photos for B. subtilis on YTD agar plate treated without and with TPP-4CB7, plot of $\zeta$-potentials of E. coli with TPP-4CB7 (PDF) 


\section{AUTHOR INFORMATION}

\section{Corresponding Author}

*E-mail: dtuncel@fen.bilkent.edu.tr.

\section{ORCID $\odot$}

Yogesh Kumar: 0000-0003-4562-3896

Dönüs Tuncel: 0000-0001-7762-9200

\section{Author Contributions}

D.T. conceived the idea and designed experiments. Y.Ku. synthesized and characterized TPP-4CB7. S.E.H. investigated singlet oxygen quantum yield. M.O. carried out further purification of TPP-4CB7, bacterial experiments, and ITC measurements. Y.Ke. performed MTT assays. D.T. and M.O. analyzed the data and wrote the manuscript. All authors have given approval to the final version of the manuscript.

\section{Notes}

The authors declare no competing financial interest.

\section{ACKNOWLEDGMENTS}

We acknowledge the financial support of The Scientific and Technological Research Council of Turkey-TÜBITAK (KBAG 215Z035).

\section{REFERENCES}

(1) Phillips, D. Light Relief: Photochemistry and Medicine. Photochem. Photobiol. Sci. 2010, 9, 1589.

(2) Wilson, B. C.; Patterson, M. S. The Physics, Biophysics and Technology of Photodynamic Therapy. Phys. Med. Biol. 2008, 53, R61.

(3) Zhou, Z.; Song, J.; Nie, L.; Chen, X. Reactive Oxygen Species Generating Systems Meeting Challenges of Photodynamic Cancer Therapy. Chem. Soc. Rev. 2016, 45, 6597.

(4) Maisch, T. Resistance in abtimicrobial photodynamic activation of bacteria. Photochem. Photobiol. Sci. 2015, 14, 1518.

(5) Maisch, T. A new strategy to destroy antibiotic resistant microorganisms: antimicrobial photodynamic treatment. Mini-Rev. Med. Chem. 2009, 9, 974.

(6) Ethirajan, M.; Chen, Y.; Joshi, P.; Pandey, R. K. The role of porphyrin chemistry in tumor imaging and photodynamic therapy. Chem. Soc. Rev. 2011, 40 (1), 340.

(7) Rajora, M. A.; Lou, J. W. H.; Zheng, G. Advancing porphyrin's biomedical utility via supramolecular chemistry. Chem. Soc. Rev. 2017, 46, 6433.

(8) Xu, L.; Liu, L.; Liu, F.; Li, W.; Chen, R.; Gao, Y.; Zhang, W. Photodynamic therapy of oligoethylene glycol dendronized reductionsensitive porphyrins. J. Mater. Chem. B 2015, 3, 3062.

(9) Singh, S.; Aggarwal, A.; Bhupathiraju, N. V. S. D. K.; Arianna, G.; Tiwari, K.; Drain, C. M. Glycosylated Porphyrins, Phthalocyanines, and Other Porphyrinoids for Diagnostics and Therapeutics. Chem. Rev. 2015, 115, 10261.

(10) Li, X.; Lee, S.; Yoon, J. Supramolecular Photosensitizers Rejuvenate Photodynamic Therapy. Chem. Soc. Rev. 2018, 47, 1174.

(11) Liu, K.; Liu, Y.; Yao, Y.; Yuan, H.; Wang, S.; Wang, Z.; Zhang, $\mathrm{X}$. Supramolecular photosensitizers with enhanced antibacterial efficiency. Angew. Chem., Int. Ed. 2013, 52, 8285.

(12) Chen, L.; Bai, H.; Xu, J.-F.; Wang, S.; Zhang, X. Supramolecular Porphyrin Photosensitizers:Controllable Disguise and Photoinduced Activation of Antibacterial Behavior. ACS Appl. Mater. Interfaces 2017, 9, 13950.

(13) Caceres, J.; Robinson-Duggon, J.; Tapia, A.; Paiva, C.; Gomez, M.; Bohne, C.; Fuentealba, D. Photochemical behavior of biosupramolecular assemblies of photosensitizers, cucurbit[n]urils and albumins. Phys. Chem. Chem. Phys. 2017, 19, 2574.

(14) El-Sheshtawy, H. S.; Chatterjee, S.; Assaf, K. I.; Shinde, M. N.; Nau, W. M.; Mohanty, J. A Supramolecular Approach for Enhanced
Antibacterial Activity and Extended Shelf-life of Fluoroquinolone Drugs with Cucurbit[7]uril. Sci. Rep. 2018, 8, 13925.

(15) Özkan, M.; Keser, Y.; Hadi, S. E.; Tuncel, D. [5]RotaxaneBased Photosensitizer for Photodynamic Therapy. Eur. J. Org. Chem. 2019, 2019, 3534.

(16) Assaf, K. I.; Nau, W. M. Cucurbiturils: From Synthesis to HighAffinity Binding and Catalysis. Chem. Soc. Rev. 2015, 44, 394.

(17) Macartney, D. H. Encapsulation of Drug Molecules by Cucurbiturils: Effects on their Chemical Properties in Aqueous Solution. Isr. J. Chem. 2011, 51, 600.

(18) Gürbüz, S.; Idris, M.; Tuncel, D. Cucurbituril-Based Supramolecular Engineered Nanostructured Materials. Org. Biomol. Chem. 2015, 13, 330.

(19) Koc, A.; Tuncel, D. Supramolecular Assemblies of Cucurbiturils with Photoactive, $\pi$-conjugated Chromophores. Isr. J. Chem. 2018, 58 , 334

(20) Khurana, R.; Kakatkar, A. S.; Chatterjee, S.; Barooah, N.; Kunwar, A.; Bhasikuttan, A. C.; Mohanty, J. Supramolecular Nanorods of (N-Methylpyridyl) Porphyrin With Captisol: Effective Photosensitizer for Anti-bacterial and Anti-tumor Activities. Front. Chem. 2019, 7, 452.

(21) Lei, W.; Jiang, G.; Zhou, Q.; Zhang, B.; Wang, X. Greatly enhanced binding of a cationic porphyrin towards bovine serum albümin by cucurbit[8] uril. Phys. Chem. Chem. Phys. 2010, 12, 13255.

(22) Koc, A.; Khan, R.; Tuncel, D. Clicked” Porphyrin-Cucurbituril Conjugate: A New Multifunctional Supramolecular Assembly Based on Triglycosylated Porphyrin and Monopropargyloxycucurbit[7]uril. Chem. - Eur. J. 2018, 24, 15550.

(23) Kumar, Y.; Patil, B.; Khaligh, A.; Hadi, S. E.; Uyar, T.; Tuncel, D. Novel Supramolecular Photocatalyst Based on Conjugation of Cucurbit[7]uril to Non-Metallated Porphyrin for Electrophotocatalytic Hydrogen Generation from Water Splitting. ChemCatChem 2019, 13, 2994.

(24) Ozkan, M.; Keser, Y.; Koc, A.; Tuncel, D. Glycosylated porphyrin-cucurbituril conjugate for photodynamic inactivation of bacteria and doxorubicin carriage for anticancer drug delivery. J. Porphyrins Phthalocyanines 2019, DOI: 10.1142/ S1088424619501578.

(25) Webber, M. J.; Langer, R. Drug Delivery by Supramolecular Design. Chem. Soc. Rev. 2017, 46, 6600.

(26) Lim, W. Q.; Yang, G.; Phua, S. Z. F.; Chen, H.; Zhao, Y. SelfAssembled Oxaliplatin(IV) Prodrug-Porphyrin Conjugate for Combinational Photodynamic Therapy and Chemotherapy. ACS Appl. Mater. Interfaces 2019, 11, 16391.

(27) Zhang, W.; Li, Y.; Sun, J.-H.; Tan, C.-P.; Ji, L.-N.; Mao, Z.-W. Supramolecular self-assembled nanoparticles for chemo-photodynamic dual therapy against cisplatin resistant cancer cells. Chem. Commun. 2015, 51, 1807.

(28) Spagnul, C.; Turner, L. C.; Boyle, R. W. Immobilized Photosensitizers for Antimicrobial Applications. J. Photochem. Photobiol., B 2015, 150, 11.

(29) Wu, X.; Zhang, Y.-M.; Liu, Y. Nanosupramolecular assembly of amphiphilic guest mediated by cucurbituril for doxorubicin delivery. RSC Adv. 2016, 6, 99729.

(30) Benyettou, F.; Fahs, H.; Elkharrag, R.; Bilbeisi, R. A.; Asma, B.; Rezgui, R.; Motte, L.; Magzoub, M.; Brandel, J.; Olsen, J.-C.; Piano, F.; Gunsalus, K. C.; Platas-Iglesias, C.; Trabolsi, A. Selective growth inhibition of cancer cells with doxorubicin-loaded $\mathrm{CB}[7]$-modified iron-oxide nanoparticles. RSC Adv. 2017, 7, 23827. 